\title{
A new look at energy release rates for quasistatically propagating cracks in inelastic materials
}

\author{
RALF PEEK $^{1}$ and XIAOMIN DENG ${ }^{2}$ \\ ${ }^{1}$ College of Engineering, University of Michigan, Ann Arbor, Michigan 48109-2125, USA \\ ${ }^{2}$ Department of Mechanical Engineering, University of South Carolina, Columbia, South Carolina 29208, USA
}

Received 1 July 1990; accepted in revised form 12 June 1992

\begin{abstract}
A mapping technique is used to derive an integral expression for the energy release rate for a quasistatically propagating crack. The derivation does not depend on any assumptions in regard to the contitutive behavior of the material. It leads to a contour integral around the crack tip, plus an area integral over the region enclosed by this contour. Only the stress and displacement fields appear in the integrands. Although for stationary crack solutions known to the authors the area integral is not convergent, for propagating crack solutions in elastoplastic material, the integrals are convergent, and lead to zero energy release rate. This confirms conclusions by Rice from an independent point of view.
\end{abstract}

\section{Notation}

Latin symbols:

a = increase in crack length, as measured from some initial location

$a(\lambda) \quad=$ location of crack at time $\lambda$

f $\quad=$ body force per unit volume

$\mathbf{u}(\mathbf{x}, \lambda)=$ displacement at location $x$ at time $\lambda$

$\mathbf{u}[\mathbf{r}, \lambda]=$ displacement vector at location $\mathbf{x}(\mathbf{r}, a(\lambda))$ at time $\lambda$

$\mathbf{u}(\mathbf{x}, a, \lambda)=$ expanded set of displacement fields defined in (7)

$\mathbf{u}^{\prime}(\mathbf{x}, \lambda)=$ incompatible displacement rate defined in (23)

$\mathbf{x}=$ position vector of point under consideration

Greek symbols:

$\hat{\lambda} \quad=$ time parameter describing the process of crack extension

$\varepsilon=$ strain tensor (small deformation theory is used)

$\rho \quad=$ distance of point under consideration from crack tip

$\sigma[\mathbf{r}, \lambda]=$ stress tensor at location $\mathbf{x}(\mathbf{r}, a(\lambda))$ at time $\lambda$

\section{Introduction}

Since Griffith's $[1,2]$ introduction of the concept of surface energy as a fracture criterion, there has been much interest in extending this concept to nonlinear as well as inelastic materials. Griffith's work is based on the observation that during the process of quasistatic crack propagation, the work done by applied loads exceeds the increase in strain energy stored in the material. To account for this deficiency, he introduced the surface energy as the energy per unit area that is required to generate a new surface by fracture of the material. The first attempts to generalize this energy balance fracture criterion to inelastic materials were made independently by Irwin [3] and Orowan [4], who proposed that the released elastic strain energy be equal to the surface energy plus the work done in plastic deformation at the crack tip. 
A general definition of this released energy was later given by Irwin [5] whose "crack extension force tendency' is now known as the strain energy release rate. The value of this energy release rate was correlated in [5] with the strength of the crack-tip elastic singular stress and displacement fields $[6,7]$. Since (under suitable conditions) these singular fields fully determine the conditions at the crack tip, this provides a rational justification for the use of energy release rates fracture criterion in linearly elastic materials. Thus the foundation of linear fracture mechanics was laid.

More generally, for elastic (nonlinear or linear) materials, the energy release rate can be evaluated conveniently by Rice's [8] path-independent $J$-integral. In a power-law elastic material, the value of the $J$-integral also uniquely characterizes the crack-tip deformation field (generally known as the HRR-field) [9, 10]. Under the assumptions of the deformation theory of plasticity, these results can also be applied to a power-law hardening elastic-plastic material. The discoveries of the path-independent $J$-integral and the HRR-field form the basis for the elastic-plastic fracture mechanics.

For a growing crack in inelastic material, the deformation theory of plasticity is not applicable. Therefore the results discussed above regarding the energy release rate and its equivalence with the $J$-integral cannot be applied. In response to this dilemma, there have been efforts to identify new integrals and to connect them to the energy release rate and/or the near-tip stress and deformation fields [11, 12]. It has been argued [13] that such crack-tip integrals are direct consequences of total energy and momentum balance.

In this paper, the subject of the energy release rates is re-visited by deriving an energy integral for a quasistatically propagating crack. The derivation employs a geometric mapping scheme by which the domain of the problem is mapped to a space in which the crack tip is stationary. An interesting feature of this integral is that time derivatives do not enter in the integrands. This novel technique appears to be advantageous over traditional methods of constructing pathindependent integrals for the energy release rate for growing cracks in that no constitutive laws need to be specified. The drawback of this technique is that its application places certain restrictions on the crack-tip singularity.

\section{Derivation of surface energy integral}

For simplicity, attention is focused on a two dimensional (plane stress or plane strain) problem. Deformations are assumed to be small, and the material homogeneous. Let the body in its undeformed configuration occupy a region $\Omega_{\mathrm{x}}$ in the $\mathbf{x}$-plane, as shown in Fig. 1 . The region $\Omega_{\mathrm{x}}$ excludes the boundaries, as well as a line along which the crack is assumed to grow. The location of the crack is described by a length parameter $a$. The region occupied by the initial surface of the body which is not part of the crack surface is denoted $\Gamma_{\mathrm{x}}$. There may be a combination of imposed displacements and applied loads on the boundary $\Gamma_{\mathbf{x}}$. However, the crack surface (both the initial crack surface as well as the surface generated by crack extension) is assumed to be traction free.

A time parameter $\lambda$ describes the process by which the applied loads are changing and the crack is extending. The applied loads may be increasing or decreasing with $\lambda$, but the process is assumed to be quasistatic. No inertia effects are included. If the material is rate independent the time parameter $\lambda$ need not necessarily be associated with real time. Any monotonically increasing parameter can be used. The natural process of quasistatic crack propagation is 


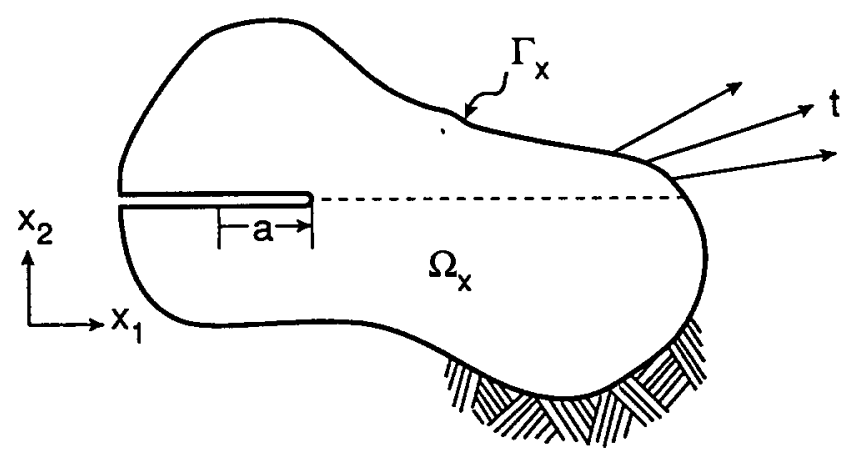

Fig. 1.

described by one parameter families of displacement and stress fields, $\mathbf{u}=\mathbf{u}(\mathbf{x}, \lambda)$ and $\sigma=\sigma(\mathbf{x}, \lambda)$ respectively, with $\mathbf{x} \in \Omega_{\mathbf{x}}$, as well as a function $a=a(\lambda)$ describing the location of the crack tip at any time.

The process of crack propagation is not one which can be established by analysis unless a crack propagation criterion is used. However, this does not prevent determination of surface energy release rates for a given process of crack propagation. The derivation of surface energy integrals employs a mapping such that there is no crack propagation for the mapped problem. At first energy release integrals are derived for an arbitrary mapping. Then attention is focused on a particular type of mapping that results in some simplification of the energy release integral.

Let $\mathbf{x}(\mathbf{r}, a)$ be a one-one family of mappings from a region $\Omega_{\mathbf{r}}$ on the $\mathbf{r}$-plane onto the region $\Omega_{\mathbf{x}}$. Conversely, the inverse family of mappings $\mathbf{r}(\mathbf{x}, a)$ maps the region $\Omega_{\mathbf{x}}$ onto the region $\Omega_{\mathrm{r}}$. This family of mappings is chosen in such a way that the location of the crack tip in the r-plane remains unchanged. Thus, the location of the crack tip in the $\mathbf{x}$-plane is $\mathbf{x}\left(\mathbf{r}_{\text {tip }}, a\right)$, where $\mathbf{r}_{\text {tip }}$ is the (constant) location of the crack tip in the r-plane. An example of such a mapping will be given later. The condition that the mappings $\mathbf{r}(., a)$ and $\mathbf{x}(., a)$ be the inverse of each other can be written as

$$
\mathbf{x}(\mathbf{r}(\mathbf{x}, a), a)=\mathbf{x} \quad \forall \mathbf{x}, a, \quad \mathbf{r}(\mathbf{x}(\mathbf{r}, a), a)=\mathbf{r} \quad \forall \mathbf{r}, a
$$

Differentiating (1a) and (1b) with respect to $\mathbf{x}$ and $\mathbf{r}$ respectively gives

$$
\mathbf{x}_{\mathbf{r}}(\mathbf{r}(\mathbf{x}, a), a) \cdot \mathbf{r}_{\mathbf{x}}(\mathbf{x}, a)=\mathbf{I} \quad \forall \mathbf{x}, a, \quad \mathbf{r}_{\mathbf{x}}(\mathbf{x}(\mathbf{r}, a), a) \cdot \mathbf{x}_{\mathbf{r}}(\mathbf{r}, a)=\mathbf{I} \quad \forall \mathbf{r}, a,
$$

where $\mathbf{I}$ is the identity tensor.

Next it is postulated that energy is dissipated by two distinguishable mechanisms:

(a) plastic straining of the material, and

(b) dissipation at the crack tip that is not predictable from continuum mechanics with local constitutive laws.

The latter component will be referred to as the surface energy $S$. To simplify the thermodynamic aspects of the problem, it is assumed, that the process of crack growth is either adiabatic (both locally and globally) or isothermal. (See [19-21] for a discussion of the thermodynamic aspects of crack growth.) Under such conditions, the time rate at which energy is being used for the 
surface formation process is

$$
S_{\lambda}(\lambda)=\int_{\Omega_{\mathbf{x}}}\left\{\mathbf{f}(\mathbf{x}, \lambda) \cdot \mathbf{u}_{\lambda}(\mathbf{x}, \lambda)-\sigma(\mathbf{x}, \lambda): \mathbf{u}_{\lambda x}(\mathbf{x}, \lambda)\right\} \mathrm{d} x_{1} \mathrm{~d} x_{2}+\int_{\Gamma_{\mathbf{x}}} \mathbf{t}(\mathbf{x}, \lambda) \cdot \mathbf{u}_{\lambda}(\mathbf{x}, \lambda)\|\mathrm{d} \mathbf{x}\| .
$$

Subscripts indicate differentiation with respect to one of the arguments. The location of the argument being referred to should be extracted from the definition of the function or field in the Notations. Thus, for example $\mathbf{u}_{a}(., .,$.$) denotes the derivative of \mathbf{u}(., .,$.$) with respect to the second$ argument, since the field $\mathbf{u}(., .,$.$) is listed in the Notations as \mathbf{u}(\mathbf{x}, a, \lambda)$. A distinction is made here between the derivative of a function or field with respect to one of its arguments (as indicated by subscripts), and partial derivatives with respect to variables that appear explicitly in an expression, for which the usual $\partial / \partial($.$) is used. The advantage of such a pedantic distinction in this$ context will become more apparent later.

Next let $\delta \mathbf{u}(\mathbf{x})$ be a virtual displacement field. From the principle of virtual displacements,

$$
\int_{\Omega_{\mathbf{x}}}\left\{\mathbf{f}(\mathbf{x}, \lambda) \cdot \delta \mathbf{u}(\mathbf{x})-\sigma(\mathbf{x}, \lambda): \delta \mathbf{u}_{x}(\mathbf{x})\right\} \mathrm{d} x_{1} \mathrm{~d} x_{2}+\int_{\Gamma_{\mathbf{x}}} \mathbf{t}(\mathbf{x}, \lambda) \cdot \delta \mathbf{u}(\mathbf{x})\|\mathrm{d} \mathbf{x}\|=0,
$$

(4) is valid for any compatible virtual displacement field. However, for $\delta \mathbf{u}(\mathbf{x})=\mathbf{u}_{\lambda}(\mathbf{x}, \lambda),(4)$ does not apply, since displacements $\mathbf{u}(\mathbf{x}, \lambda)$ involve crack extension. Therefore $\mathbf{u}_{\lambda}(\mathbf{x}, \lambda)$ is not a compatible displacement field. Indeed if $\mathbf{u}(\mathbf{x}, \lambda)$ were compatible, the surface energy computed from (3) would always be zero.

To simplify the right hand side of (3), the field $\mathbf{u}_{\lambda}(\mathbf{x}, \lambda)$ will be decomposed into a compatible part for which the contribution of the integrals is zero by the principle of virtual work, and an incompatible part, which gives rise to the surface energy. For this purpose, we define fields of displacements, stresses, etc. on the r-plane as follows

$$
\mathbf{u}[\mathbf{r}, \lambda]=\mathbf{u}(\mathbf{x}(\mathbf{r}, a(\lambda)), \lambda), \quad \sigma[\mathbf{r}, \hat{\lambda}]=\sigma(\mathbf{x}(\mathbf{r}, a(\hat{\lambda})), \hat{\lambda}), \quad \text { etc. }
$$

Note that $\mathbf{u}(.$, .) and $\mathbf{u}[.,$.$] represent different vector valued functions. By replacing \mathbf{r}$ in the first of (5) by $\mathbf{r}(\mathbf{x}, a(\lambda))$ and making use of (1a) it is seen that

$$
\mathbf{u}(\mathbf{x}, \lambda)=\mathbf{u}[\mathbf{r}(\mathbf{x}, a(\lambda)), \lambda]
$$

At this point an expanded two-parameter set of displacement fields can be defined as

$$
\mathbf{u}(\mathbf{x}, a, \lambda) \equiv \mathbf{u}[\mathbf{r}(\mathbf{x}, a), \lambda]
$$

Clearly, from (6) and (7),

$$
\mathbf{u}(\mathbf{x}, a(\lambda), \lambda)=\mathbf{u}(x, \lambda)
$$

This means that along the line $a=a(\lambda)$ the expanded displacement fields coincide with real displacement fields for the crack propagation process. On the other hand, for $a \equiv a\left(\lambda_{0}\right)<a(\lambda)$, $\mathbf{u}(\mathbf{x}, a, \lambda)$ represents the displacement field at time $\lambda$ mapped back to the crack location at some 
earlier time $\lambda_{0}$. Specifically this displacement field embodies the crack tip field of time $\lambda$ at the location of the crack at time $\lambda_{0}$.

If $a$ is kept fixed and $\lambda$ is increased, the expanded displacement field involves displacements that are changing, but always mapped back to the same crack location. This process does not involve crack extension. Therefore $\mathbf{u}_{\lambda}(\mathbf{x}, a, \lambda)$ is a compatible displacement field

If, on the other hand, $\lambda$ is kept fixed and $a$ is increased, the displacements at any point $\mathbf{r} \in \Omega_{\mathrm{r}}$ remain constant but they are mapped to a geometry involving an extending crack. Therefore two arbitrarily close points might be separated as the crack propagates between them. This means that $\mathbf{u}_{a}(\mathbf{x}, a, \lambda)$ represents an incompatible displacement field.

The above remarks form the basis for the decomposition of $\mathbf{u}_{\lambda}(\mathbf{x}, \lambda)$ into incompatible and compatible portions, as follows

$$
\mathbf{u}_{\lambda}(\mathbf{x}, \lambda)=\frac{\partial}{\partial \lambda} \mathbf{u}(\mathbf{x}, a(\lambda), \lambda)=\mathbf{u}_{a}(\mathbf{x}, a(\lambda), \lambda) a_{\lambda}(\lambda)+\mathbf{u}_{\lambda}(\mathbf{x}, a(\lambda), \lambda)
$$

The first and second terms on the right hand side are incompatible and compatible portions respectively. In substituting (9) into the expression for the surface energy rate, (3), only the incompatible part needs to be used, since the compatible part satisfies the principle of virtual displacements, (4)*. This yields the following rate of surface energy per unit length of crack extension

$$
G \equiv \frac{S_{\lambda}(\lambda)}{a_{\lambda}(\lambda)}=\int_{\Omega_{\mathbf{x}}}\left\{\mathbf{f}(\mathbf{x}, \lambda) \cdot \mathbf{u}^{\prime}(\mathbf{x}, \lambda)-\sigma(\mathbf{x}, \lambda): \mathbf{u}_{\mathbf{x}}^{\prime}(\mathbf{x}, \lambda)\right\} \mathrm{d} x_{1} \mathrm{~d} x_{2}+\int_{\Gamma_{\mathbf{x}}} \mathbf{t}(\mathbf{x}, \lambda) \cdot \mathbf{u}^{\prime}(\mathbf{x}, \lambda)\|\mathrm{d} \mathbf{x}\|,
$$

where

$$
\mathbf{u}^{\prime}(\mathbf{x}, \lambda) \equiv \mathbf{u}_{a}(\mathbf{x}, a(\lambda), \lambda)
$$

will be referred to as the incompatible displacement rate. The expression on the right hand side can be evaluated as follows

$$
\mathbf{u}^{\prime}(\mathbf{x}, \lambda)=\left\{\frac{\partial}{\partial a} \mathbf{u}[\mathbf{r}(\mathbf{x}, a), \lambda]\right\}_{a=a(\lambda)}=\mathbf{u}_{\mathbf{r}}[\mathbf{r}(\mathbf{x}, a(\lambda)), \lambda] \cdot \mathbf{r}_{a}(\mathbf{x}, a(\lambda)) .
$$

To further simplify this expression for the incompatible displacement rate, differentiate (1a) with respect to $a$ and replace $a$ by $a(\lambda)$ to obtain

$$
\mathbf{r}_{a}(\mathbf{x}, a(\lambda))=-\left\{\mathbf{x}_{\mathbf{r}}(\mathbf{r}(\mathbf{x}, a(\lambda)), a(\lambda))\right\}^{-1} \cdot \mathbf{x}_{a}(\mathbf{r}(\mathbf{x}, a(\lambda)), a(\lambda))
$$

Differentiating (6) with respect to $\mathbf{x}$, and making use of (2a) gives

$$
\mathbf{u}_{\mathbf{r}}[\mathbf{r}(\mathbf{x}, a(\lambda)), \lambda]=\mathbf{u}_{\mathbf{x}}(\mathbf{x}, \lambda) \cdot \mathbf{x}_{\mathbf{r}}((\mathbf{r}(\mathbf{x}, a(\lambda)), a(\lambda))
$$

\footnotetext{
* It can readily be verified that for crack fields in which the products of stress times displacement gradient behave like $1 / \rho$, the integrals in (4) are convergent for $\delta \mathbf{u}(\mathbf{x})=\mathbf{u}_{\lambda}(\mathbf{x}, a(\lambda), \lambda)$. Therefore it is legitimate to apply the principle of virtual work.
} 
Substituting (13) and (14) into (12) yields

$$
\mathbf{u}^{\prime}(\mathbf{x}, \hat{\lambda})=-\mathbf{u}_{\mathbf{x}}(\mathbf{x}, \lambda) \cdot \mathbf{x}_{a}(\mathbf{r}(\mathbf{x}, a(\lambda)), a(\lambda))
$$

The gradient of the incompatible displacement rate is also required in the surface energy integral, (10), and is given by

$$
\begin{aligned}
\mathbf{u}_{\mathbf{x}}^{\prime}(\mathbf{x}, \lambda)= & -\mathbf{u}_{\mathbf{x x}}(\mathbf{x}, \lambda) \cdot \mathbf{x}_{a}(\mathbf{r}(\mathbf{x}, a(\lambda)), a(\lambda)) \\
& -\mathbf{u}_{\mathbf{x}}(\mathbf{x}, \lambda) \cdot \mathbf{x}_{a r}(\mathbf{r}(\mathbf{x}, a(\lambda)), a(\lambda)) \cdot\left\{\mathbf{x}_{\mathbf{r}}(\mathbf{r}(\mathbf{x}, a(\lambda)), a(\lambda))\right\}^{-1}
\end{aligned}
$$

Substituting (15) and (16) into (10) yields the desired expression for the energy release rate. Integrability conditions will be discussed later, in the context of a specific class of mappings. It is interesting to note that the surface energy depends only on the current state of stresses and displacements. Time derivatives do not enter into the expression. Derivatives with respect to the mapping parameter $a$ are taken only for the mapping functions themselves. These can readily be evaluated if the mapping $\mathbf{x}(\mathbf{r}, a)$ is available in explicit form.

Equations (10), (15), and (16) define the surface energy for an arbitrary mapping. In order to simplify this result, attention is now focused on a particular class of mappings. For this purpose, let region $\Omega_{\mathrm{r}}$ be identical to region $\Omega_{\mathrm{x}}$, and also let the position of the crack tip $\mathbf{r}_{\text {tip }}$ in the r-plane coincide with the location of the crack tip in the $\mathbf{x}$-plane, at the current time, $\lambda$. Without loss of generality we may assume that this location of the crack tip is described by a value of the crack extension parameter of

$$
a(\lambda)=0
$$

Define a contour $C$ in $\Omega_{\mathrm{r}}$ that encloses the crack tip but is otherwise arbitrary (as for the $J$-integral). A second contour $C^{\prime}$ encloses the contour $C$ as well as the crack tip, and is placed a constant distance $b$ away from the contour $C$, as shown in Fig. 2 . The distance $b$ is measured perpendicular to the contour $C$. A unit vector that is normal to $C$ and pointing towards $C^{\prime}$ will be denoted by $\mathbf{n}$. Thus the contour $C^{\prime}$ could be described as the set of all $\mathbf{x}$ such that $\mathbf{x}=\mathbf{y}+b \mathbf{n}$ where $\mathbf{y} \in C$. The region around the crack tip up to the contour $C$ is denoted $\Omega$; the region between contours $C$ and $C^{\prime}$ is denoted $\Omega_{1}$; and what remains of the region $\Omega_{\mathrm{r}}$ is denoted $\Omega_{2}$. All these definitions are illustrated in Fig. 2.

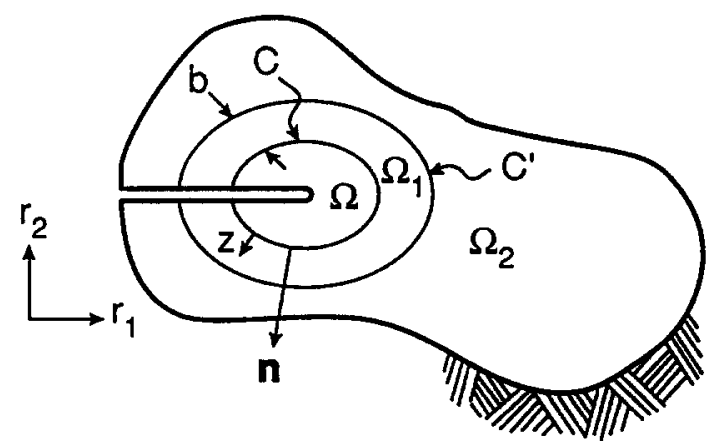

Fig. 2. 
The mapping $\mathbf{x}(\mathbf{r}, a)$ is defined as follows

$$
\begin{aligned}
\mathbf{x}(\mathbf{r}, a) & =\mathbf{r}+a \mathbf{e}_{1} & & \text { for } \mathbf{r} \in \Omega, \\
& =\mathbf{r}+\frac{b-z}{b} a \mathbf{e}_{1} & & \text { for } \mathbf{r} \in \Omega_{1}, \\
& =\mathbf{r} & & \text { for } \mathbf{r} \in \Omega_{2},
\end{aligned}
$$

where $\mathbf{e}_{1}$ is the unit vector in the $x_{1}$ direction, which is assumed to be parallel to the direction of crack growth, and $z$ is the distance of the point under consideration to the contour $C$, measured perpendicular to the contour. From the latter definition, it is apparent that

$$
\frac{\partial z}{\partial \mathbf{r}}=\mathbf{n}
$$

Thus the required derivatives of the mapping function are

$$
\begin{aligned}
\mathbf{x}_{\mathbf{r}}(\mathbf{r}, a) & =\mathbf{I} & & \text { for } \mathbf{r} \in \Omega, \\
& =\mathbf{I}-\frac{a}{b} \mathbf{e}_{1} \mathbf{n} & & \text { for } \mathbf{r} \in \Omega_{1}, \\
& =\mathbf{I} & & \text { for } \mathbf{r} \in \Omega_{2} ; \\
\mathbf{x}_{a}(\mathbf{r}, a) & =\mathbf{e}_{1} & & \text { for } \mathbf{r} \in \Omega \\
& =\frac{b-z}{b} \mathbf{e}_{1} & & \text { for } \mathbf{r} \in \Omega_{1} \\
& =\mathbf{0} & & \text { for } \mathbf{r} \in \Omega_{2} \\
\mathbf{x}_{a \mathbf{r}}(\mathbf{r}, a) & =\mathbf{0} & & \text { for } \mathbf{r} \in \Omega_{1} \\
& =-\frac{1}{b} \mathbf{e}_{1} \mathbf{n} & & \text { for } \mathbf{r} \in \Omega_{1} \\
& =\mathbf{0} & & \text { for } \mathbf{r} \in \Omega_{2}
\end{aligned}
$$

Note that all these derivatives are integrable, even though the derivatives with respect to $\mathbf{r}$ strictly do not exist at the contours $C$ and $C^{\prime}$.

At this point the derivatives of the mapping functions can be evaluated at $a=a(\lambda)$ given by (17), and the results can be substituted into the expressions for the incompatible displacement rate and its gradient, (15) and (16), to obtain

$$
\begin{aligned}
\mathbf{u}^{\prime}(\mathbf{x}, \lambda) & =-\mathbf{u}_{\mathbf{x}}(\mathbf{x}, \lambda) \cdot \mathbf{e}_{1} & & \text { for } \mathbf{x} \in \Omega, \\
& =-\frac{b-z}{b} \mathbf{u}_{\mathbf{x}}(\mathbf{x}, \lambda) \cdot \mathbf{e}_{1} & & \text { for } \mathbf{x} \in \Omega_{1}, \\
& =\mathbf{0} & & \text { for } \mathbf{x} \in \Omega_{2} ;
\end{aligned}
$$




$$
\begin{aligned}
\mathbf{u}_{\mathbf{x}}^{\prime}(\mathbf{x}, \lambda) & =-\mathbf{u}_{\mathbf{x x}}(\mathbf{x}, \lambda) \cdot \mathbf{e}_{1} & & \text { for } \mathbf{x} \in \mathbf{\Omega}, \\
& =-\frac{b-z}{b} \mathbf{u}_{\mathbf{x x}}(\mathbf{x}, \lambda) \cdot \mathbf{e}_{1}+\frac{1}{b} \mathbf{u}_{\mathbf{x}}(\mathbf{x}, \lambda) \cdot \mathbf{e}_{\mathbf{1}} \mathbf{n} & & \text { for } \mathbf{x} \in \Omega_{1}, \\
& =\mathbf{0} & & \text { for } \mathbf{x} \in \Omega_{2} .
\end{aligned}
$$

Substituting the last two expressions into the surface energy integral, (10), and taking the limit as $b \rightarrow 0$ gives

$$
\begin{aligned}
G= & \int_{\Omega}\left\{-\mathbf{f}(\mathbf{x}, \lambda) \cdot \mathbf{u}_{\mathbf{x}}(\mathbf{x}, \lambda) \cdot \mathbf{e}_{1}+\sigma(\mathbf{x}, \lambda): \mathbf{u}_{\mathbf{x}}(\mathbf{x}, \lambda) \cdot \mathbf{e}_{1}\right\} \mathrm{d} x_{1} \mathrm{~d} x_{2} \\
& -\int_{C} \sigma(\mathbf{x}, \lambda): \mathbf{u}_{\mathbf{x}}(\mathbf{x}, \lambda) \cdot \mathbf{e}_{1} \mathbf{n}\|\mathrm{d} \mathbf{x}\| .
\end{aligned}
$$

In this final result it is apparent that the pedantic notation in which all function dependencies are indicated explicitly can safely be abandoned, leading to restatement of (25) in the following form

$$
G=\int_{\Omega}\left\{-\mathbf{f} \cdot \frac{\partial \mathbf{u}}{\partial x_{1}}+\sigma: \frac{\partial \varepsilon}{\partial x_{1}}\right\} \mathrm{d} x_{1} \mathrm{~d} x_{2}-\int_{C} \mathbf{t} \cdot \frac{\partial \mathbf{u}}{\partial x_{1}}\|\mathrm{~d} \mathbf{x}\|
$$

where

$$
\varepsilon=\frac{1}{2}\left[\frac{\partial \mathbf{u}}{\partial \mathbf{x}}+\left(\frac{\partial \mathbf{u}}{\partial \mathbf{x}}\right)^{T}\right], \quad \mathbf{t}=\sigma \cdot \mathbf{n}
$$

are the strain tensor, and the traction acting on the surface that would be formed by cutting the body along the contour $C$, respectively.

\section{Discussion}

If there are no body forces, and the material is elastic with a strain energy density function $U(\varepsilon)$, (26) reduces to

$$
G=\int_{\Omega} \frac{\partial U}{\partial x_{1}} \mathrm{~d} x_{1} \mathrm{~d} x_{2}-\int_{C} \mathbf{t} \cdot \frac{\partial \mathbf{u}}{\partial x_{1}}\|\mathrm{~d} \mathbf{x}\| .
$$

If the integral over $\Omega$ is convergent so that Green's theorem can be applied, this reduces to

$$
G=\int_{C} U \mathrm{~d} x_{2}-\int_{C} \mathbf{t} \cdot \frac{\partial \mathbf{u}}{\partial x_{1}}\|\mathrm{~d} \mathbf{x}\|,
$$

which coincides with Rice's expression for the $J$-integral. 
The advantage of the present integral, (25), over the $J$-integral is that it is valid for any material, not just elastic materials, provided that the integrals are convergent. The crack tip field must be that for a propagating crack, since the assumption of a propagating crack is inherent to the present derivation of the surface energy integral. For inelastic materials the crack tip fields are strongly dependent on whether they are formed by crack propagation or by loading of a material with a preexisting crack.

A drawback of the present integrals, (26), is that they are not always convergent. If there exist positive constants, $M_{\Omega 1}, M_{\Omega 2}, M_{C}, \alpha$ and $\beta$ such that

$$
\left|\mathbf{f} \cdot \frac{\partial \mathbf{u}}{\partial x_{1}}\right| \leqslant M_{\Omega 1} \rho^{-2+\alpha},\left|\sigma: \frac{\partial \varepsilon}{\partial x_{1}}\right| \leqslant M_{\Omega 2} \rho^{-2+\beta},\left|\mathbf{t} \cdot \frac{\partial \mathbf{u}}{\partial x_{1}}\right| \leqslant M_{C} \rho^{-1},
$$

for all $\mathbf{x} \in \Omega_{\mathbf{x}}$, then it can be verified (by choosing the $C$ to be a circle centered at the crack tip) that the integrals, (26), are convergent. However, (30b) is not satisfied for all elastic and elastoplastic stationary crack solutions the authors are aware of. For example, for the linearly elastic case, the product of a stress times the strain gradient behaves like $1 / \rho^{2}$ as the crack tip is approached. It also can be shown after some algebra that for the linear elastic crack tip field, the Cauchy principal value of the integral over $\Omega$ (obtained by excluding an arbitrarily small circle at the crack tip) exists for both the plane strain and the plane stress cases, and is zero, if $\Omega$ is a circle centered at the crack tip. The integral over $C$ is nonzero, but its value is less than the energy release rate (except for the incompressible case in plane strain). Thus while the Cauchy principal value of the integral over $\Omega$ exists for the linearly elastic case it does not lead to the correct expression for the energy release rate. This is not unexpected since the integrals are not convergent, and there is no justification for excluding a circular region at the crack tip from the domain of integration, no matter how small this circular region may be.

From the above observations it is seen that the surface energy integral, (26), fails for typical stationary crack-tip fields. However, for quasistatically propagating cracks in elastic-plastic solids, the crack-tip fields possess singularities [14-16] which satisfy the inequalities specified in (30). In such cases, the integrals are convergent and the energy release rate from (26) is found to be zero, in agreement with the conclusions of Rice [17,18] who suggested [18] that this paradox can be attributed to the negligence of the effects of the crack-tip fracture process zone on the surrounding continuum fields. Indeed, by taking the contour $\mathrm{C}$ to be a circle centered at the crack tip, and taking the limit as the radius of this circle becomes vanishingly small, the energy release rate is found to be zero whenever there exist positive constants $M_{\Omega 1}, M_{\Omega 2}, M_{C}, \alpha, \beta$, and $\gamma$ such that the following inequalities apply for all $\mathbf{x} \in \Omega_{\mathbf{x}}$ :

$$
\left|\mathbf{f} \cdot \frac{\partial \mathbf{u}}{\partial x_{1}}\right| \leqslant M_{\Omega 1} \rho^{-2+\alpha}, \quad\left|\sigma: \frac{\partial \varepsilon}{\partial x_{1}}\right| \leqslant M_{\Omega 2} \rho^{-2+\beta}, \quad\left|\mathbf{t} \cdot \frac{\partial \mathbf{u}}{\partial x_{1}}\right| \leqslant M_{\mathbf{C}} \rho^{-1+\gamma} .
$$

\section{Conclusions}

It is postulated that the process of quasistatic crack propagation in an inelastic (history dependent) material involves two distinct mechanisms of energy dissipation: (a) plastic straining of the material, and (b) dissipation at the crack tip that is not predictable from continuum 
mechanics with local constitutive laws. Energy dissipation for mechanism (b) is proportional to the new surface area generated by fracture. Therefore it is referred to as the surface energy. Using a mapping technique, an integral expression for the surface energy, (26), is derived that is valid for inelastic as well as elastic materials, provided that the integrals are convergent.

As for the $J$-integral a contour surrounding the crack tip is defined, and the result is independent of the choice of this contour. In this case however, the expression for the energy release rate includes a domain integral over the region enclosed by the contour, as well as a contour integral.

An interesting feature of the surface energy integrals is that they depend only on current stress and displacement fields. Time derivatives do not enter in the integrands. However the solution for stresses and displacements must be derived for a propagating crack, since the definition of the surface energy for inelastic materials hinges on the assumption of a propagating crack.

Sufficient conditions for convergence of the surface energy integrals are shown in (30). The first of these conditions is not satisfied for all stationary crack tip fields known to the authors. However, for a propagating crack in elastoplastic-material, the integrals are convergent and lead to a zero surface energy release rate. This confirms conclusions by Rice $[17,18]$ from an independent point of view.

\section{Acknowledgements}

The authors are grateful for some stimulating discussions on the topic of this paper with Ares $\mathbf{J}$. Rosakis. Funding was provided in part by the National Science Foundation under Grant No. CES-8857002.

\section{References}

1. A.A. Griffith, Transactions, Royal Society of London, Series A 221 (1920) 163-198.

2. A.A. Griffith, Proceedings, First International Congress of Applied Mechanics, Delft (1924).

3. G.R. Irwin, Fracturing of Metals, American Society for Metals, Cleveland (1948).

4. E. Orowan, Report of Progress in Physics 12 (1949).

5. G.R. Irwin, in Sagamore Conference Proceedings, Vol. II, Syracuse University Press (1956) 289-305.

6. G.R. Irwin, Journal of Applied Mechanics 24 (1957) 361-364.

7. M.L. Williams, Journal of Applied Mechanics 24 (1957) 109-114.

8. J.R. Rice, Journal of Applied Mechanics 35 (1968) 279-386.

9. J.W. Hutchinson, Journal of the Mechanics and Physics of Solids 16 (1968) 13--31.

10. J.R. Rice an G.F. Rosengren. Journal of the Mechanic's and Physics of Solids 16 (1968) 1-12.

11. S. Aoki. K. Kishimoto and M. Sakata, Journal of Applied Mechanics 48 (1981) 825-829.

12. L.B. Freund and J.W. Hutchinson. Journal of the Mechanics and Physics of Solids 33 (1985) 169-191.

13. B. Moran and C.F. Shih. Engineering Fracture Mechancs 27 (1987) 615-642.

14. J.C. Amazigo and J.W. Hutchinson. Journal of the Mechanics and Physics of Solids 25 (1977) 81-97.

15. Y.C. Gao and K-C. Hwang, in Proceedings, 5th International Congress of Fracture 2 (1981) 669-682.

16. J.R. Rice, Mechanics of Solids. H.G. Hopkins et al. (eds.), (1982) 539--562.

17. J.R. Rice, in Proceedings, Ist International Congress of Fracture 1 (1966) 309-340.

18. J.R. Rice, in Proceedings, 8th U.S. National Congress of Applied Mechanics, R.E. Kelly (ed.), Western Periodicals, North Hollywood, California (1979) 191-216.

19. J. Kestin, A Course in Thermodynamics, Vol. II, Hemisphere Publishing (1979).

20. Q.S. Nguyen. in Advances in Fracture Research. D. Francois et al. (eds.), (1981) 2179-2185.

21. Q.S. Nguyen, in Three-Dimensional Constitutice Relations and Ductile Fracture, S. Nemat Nasser (ed.), (1981) 315-330. 